ARTíCULOS DE INVESTIGACIÓN

\title{
Clases textuales de la formación en derecho: Descripción y orientaciones para su enseñanza
}

\author{
Classes textuais de formação em direito: Descrição e orientações para seu ensino
}

Textual classes in law education: Description and guidelines for teaching

\author{
Paulina Meza \\ Universidad de La Serena, Chile
}

Felipe González-Catalán

Pontificia Universidad Católica de Valparaíso, Chile

\author{
Allison Pastén \\ Universidad Católica del Norte, Chile
}

\author{
Maximiliano Barahona \\ Pontificia Universidad Católica de Valparaíso, Chile
}

RESUMEN El objetivo de esta investigación es identificar y caracterizar las clases textuales de modalidad escrita producidas por estudiantes de derecho en dos universidades chilenas y, sobre la base de ello, presentar dos propuestas didácticas para trabajar las clases. En el marco de una investigación cualitativa, diseñamos y aplicamos entrevistas a profesores que ejercen la docencia en las instituciones estudiadas con el fin de reconocer las clases textuales trabajadas en la formación de abogados. Como resultados centrales, identificamos las clases textuales producidas por los estudiantes de derecho. Además, proponemos una taxonomía para clasificar y caracterizar las clases registradas. En función de lo anterior, desarrollamos dos propuestas para la enseñanza de dichas clases: una general, a nivel de familias de clases textuales, y otra específica, a nivel de cada clase textual. En conclusión, nuestro trabajo constituye un avance en la superación de algunos vacíos en el ámbito de la enseñanza de la escritura jurídica.

PALABRAS CLAVE Alfabetización, derecho y jurisprudencia, didácticas específicas, educación superior, formación profesional, materiales didácticos, textos. 
RESUMO O objetivo desta pesquisa é identificar e caracterizar as classes textuais da modalidade escrita produzidas por estudantes de direito em duas universidades chilenas e, com base nisso, apresentar duas propostas didáticas para trabalhar nessas classes. No âmbito de uma investigação qualitativa, elaboramos e aplicamos entrevistas com professores que lecionam nas instituições estudadas, a fim de reconhecer as classes textuais trabalhadas na formação de advogados. Como resultados centrais, identificamos as classes textuais produzidas pelos estudantes de Direito; além disso, propomos uma taxonomia para classificar e caracterizar as classes registradas. Com base no exposto, desenvolvemos duas propostas para o ensino de tais classes: uma geral, no nível das famílias das classes textuais; e outra, específica, no nível de cada classe textual. Concluindo, nosso trabalho constitui um avanço na superação de algumas lacunas no campo da educação em redação jurídica.

PALAVRAS-CHAVE Alfabetização, direito e jurisprudência, didática específica, ensino superior, formação profissional, materiais didáticos, textos.

ABSTRACT This research aims to identify and characterize the written textual classes produced by law students during their training at two Chilean universities and, on this basis, to present two didactic proposals to approach such textual classes. Framed in qualitative research, we designed an interview for professors who taught in specific institutions in order to recognize the textual classes used in the training of lawyers. As central results, we identify the main textual classes produced by law students; and, in addition, we propose a taxonomy to classify and characterize the registered classes. Based on this, we developed two proposals for teaching such classes: a general one, at the level of families of textual classes; and a specific one, at the level of each textual class. In conclusion, our work is a breakthrough in overcoming some gaps in the field of teaching legal writing.

KEYWORDS Literacy, law and jurisprudence, specific didactics, higher education, professional training, didactic materials, texts.

\section{Introducción}

Las investigaciones empíricas en el campo de la escritura jurídica aún son sumamente escasas. Los pocos estudios que se han desarrollado en español, hasta ahora, responden principalmente a una caracterización de los géneros discursivos de mayor uso en dicho ámbito (Agüero, 2014; Aguilar, 2017; entre otros). Estas propuestas han sido abordadas desde una mirada primordialmente lingüística, en la que la visión del especialista en derecho pasa a un segundo plano o, incluso, no es considerada. Otros trabajos muy recientes describen cómo los abogados perciben la escritura, concretamente la escritura clara en el ámbito jurídico (Meza y otros, 2020), sin embargo, dados los objetivos de la investigación, no se desarrolla un análisis de textos jurídicos. 
En otros ámbitos cercanos al derecho, tenemos interesantes avances como, por ejemplo, la identificación de los géneros más frecuentes y dificultosos de escribir por parte de especialistas, estudiantes y legos de la administración (da Cunha y Montané, 2020). En esta línea, se ha diseñado Artext, un sistema automático de ayuda a la redacción de textos de esta misma esfera y para el mismo tipo de usuarios mencionados (da Cunha, Montané e Hysa, 2017). Sin embargo, el ámbito de la administración no corresponde exactamente a la formación de abogados ni al ejercicio del derecho.

En el caso de Chile, país al que pertenecen los datos recolectados en este trabajo, algunas investigaciones recientes (Agüero, 2014; Aguilar, 2017) tienen como propósito central la construcción de una propuesta teórica en relación con la descripción de géneros y macrogéneros discursivos de la comunidad jurídica (Agüero, 2014), haciéndose cargo del fenómeno desde una perspectiva lingüística teórica. Todo esto en tanto los avances referidos al desarrollo de una clasificación de los géneros discursivos se han concentrado en caracterizar su función en el ámbito jurídico (Aguilar, 2017), sin avanzar en la determinación de la utilidad de esa caracterización ni incorporar orientaciones que apuesten por una didáctica de la escritura en la formación de abogados. Además, en términos generales, las investigaciones existentes centran sus propuestas en el concepto de género (Agüero, 2014; Aguilar, 2017), que muchas veces resulta complejo de aplicar, incluso para especialistas en lengua, que confunden géneros discursivos con tipos textuales, como es el caso de Aguilar (2017). Es importante mencionar que es aún más complejo para quienes no son especialistas en dicha área, como abogados que pretendan abordar la producción de textos en sus cursos o estudiantes de derecho que requieran de una guía para elaborar sus trabajos, ya que la noción de género discursivo proviene de la teoría lingüística especializada.

Ahora, en cuanto a los trabajos sobre didáctica de la escritura en la universidad, contamos con diversas iniciativas cuyo propósito central es entregar herramientas específicas a los estudiantes con el fin de que logren adaptarse a un nuevo contexto formativo y, por tanto, a los requerimientos de la nueva comunidad discursiva a la que ingresan (Fallahi y otros, 2009; Ortiz, 2015; Moyano, 2018; entre muchos otros). Sin embargo, la mayor parte de las propuestas buscan dar respuesta a las problemáticas heredadas desde los estudios previos, por tanto, se transforman en cursos remediales de carácter genérico que podrían ser aplicados en cualquier campo del saber.

Más específicamente, en el marco de la didáctica de la escritura en derecho, se han presentado algunas propuestas generales que, por ejemplo, abordan la formación en competencias comunicativas de estudiantes de primer año (Sologuren, Bonifaz y Núñez, 2019). Sin embargo, se trata de una propuesta general que podría ser utilizada en cualquier disciplina y, además, se plantea desde un enfoque exclusivamente lingüístico en el que no se considera la perspectiva de los miembros de la comunidad disciplinar jurídica. 
La predominancia del enfoque lingüístico en la sistematización y caracterización de los textos de la comunidad jurídica es concordante con lo observado en las salas de clase en las que se forman los futuros abogados. En ellas, y específicamente en las asignaturas orientadas al desarrollo y fortalecimiento de la competencia escritural de la disciplina y de las habilidades comunicativas en general, es habitual verificar que los profesores a cargo son especialistas en el área de la lengua, que desconocen los requerimientos específicos de la disciplina, o son abogados que no poseen formación explícita en escritura ni en su didáctica. Esto deviene en dos tipos de enseñanza y evaluación de la escritura en las disciplinas: una general y mucho más extendida, que la concibe como una habilidad transferible a diferentes contextos y que, en consecuencia, es enseñada y evaluada por profesores de lengua; y otra disciplinar, que la entiende como una competencia altamente específica, propia de diferentes comunidades, por lo que es instruida por profesores de alguna especialidad determinada. ${ }^{1}$

Por todo lo anterior, y con la intención de avanzar a un conocimiento más aplicado en el campo de la escritura jurídica y su enseñanza, es que en este estudio proponemos un objetivo doble. Por una parte, identificar las clases textuales de modalidad escrita producidas por estudiantes de derecho durante su formación en dos universidades chilenas a partir del discurso de los docentes y, por otra, presentar dos propuestas didácticas de carácter interdisciplinario para el desarrollo de la competencia escritural de abogados en formación sobre la base de los resultados descriptivos. Para esto, desarrollamos un trabajo de corte cualitativo (Maxwell, 2012; Creswell y Creswell, 2017) y un diseño fenomenológico (Bisquerra, 2009).

Creemos que una propuesta como la que presentamos es necesaria, ya que la escritura no solo es una de las actividades más complejas y exigentes en la educación superior (Vargas, 2011), sino también una de las más importantes, toda vez que está estrechamente vinculada al éxito en casi todos los aspectos de la vida (Graham, 2018). Así, se ha sugerido que la carencia de habilidades de escritura a nivel universitario constituye una de las causas de reprobación, retraso y deserción (Cristina, 2010). Además, en Chile se ha detectado una tendencia al alza en la deserción universitaria en los últimos años (González Catalán y Arismendi, 2018; Rodríguez y otros, 2018). A lo anterior debemos sumar que las competencias necesarias para dominar los modos de leer y escribir en la universidad no se adquieren de forma espontánea, sino que requieren de un proceso instruccional intencionado (Castelló, 2009). Además, la competencia para comunicarse por escrito de forma adecuada a los requerimientos de cada profesión no es fácil de alcanzar y no siempre se enseña de manera explícita durante la formación de pregrado (González, Meza y Castellón, 2019). Lo anterior es

1. Paulina Meza, «Caracterización discursiva de géneros producidos por estudiantes de derecho y Medicina: Su relación con la percepción de la autoeficacia en la escritura, la calidad general del texto y la evaluación disciplinar», 2017, disponible en bit.ly/2BoVESi. 
aún más relevante en el caso de los estudiantes de derecho, ámbito en el que la escritura es una herramienta fundamental, por lo que la forma en la que alguien escriba importa tanto como lo que escribe (López, 2011) y donde, además, la precisión léxica y retórica tienen un rol central (Aguilar, 2017). En este sentido, nuestra propuesta puede ayudar a que distintos miembros de la comunidad académica jurídica (estudiantes, docentes de derecho y de lengua, abogados noveles, entre otros) puedan trabajar en el desarrollo de habilidades de escritura especializada y, así, facilitar la integración de los estudiantes de forma competente a su comunidad discursiva.

\section{Antecedentes teóricos}

Nuestra investigación se basa en dos conceptos fundamentales: producción de textos académicos en derecho y clase textual. Estos serán los que desarrollaremos a continuación.

\section{La producción de textos académicos en derecho}

La escritura en la universidad es una actividad compleja que requiere de una serie de competencias que van más allá de lo que un estudiante aprende en su formación en enseñanza básica y media (Castelló, 2009). Esta dificultad aumenta exponencialmente si se circunscribe a un área específica del conocimiento, máxime si se trata de derecho, en la que el dominio de las habilidades comunicativas es fundamental (Meza y González-Catalán, en prensa).

En cuanto a los textos académicos producidos en derecho, investigaciones recientes indican que los abogados en formación producen exclusivamente textos jurídicos (Aguilar, 2017), que se definen como «todo escrito relacionado con las normas legales o con la administración de la justicia» (Muñoz, 2017: 20). Para caracterizar este tipo de textos se han utilizado criterios como: macroestructura, contenido y fuente $(\mathrm{Mu}-$ ñoz, 2017). Desde nuestra perspectiva, la afirmación de Aguilar (2017) es restringida y parcialmente correcta, ya que no da cuenta de la variedad de textos que producen los estudiantes de derecho a lo largo de su formación. Por ello, en esta investigación utilizaremos el concepto «textos académicos de la formación en derecho», que incluye tanto los textos disciplinares (sentencia, contrato, demanda, etcétera) como también los transversales a la educación universitaria (ensayos, pruebas, informes, etcétera) y que, por cierto, contribuyen a su formación académica.

\section{Clase textual}

El concepto «clase textual», proveniente de la lingüística del texto, hace referencia a un tipo de clasificación empírica de los textos por parte de los usuarios. Ella es realizada por los miembros de una comunidad lingüística, es decir, se trata de clasificacio- 
nes cotidianas que pueden mencionarse por medio de determinados lexemas sobre cierta clase textual. Por ejemplo, «esto es un cuento», «esto es un artículo», etcétera (Ciapuscio, 1998). Dicho de otro modo, una clase textual corresponde a la denominación natural que los usuarios dan a un determinado texto.

Las clases textuales se han desarrollado históricamente dentro de la comunidad lingüística y forman parte del saber cotidiano de los hablantes. Esta última característica es la que diferencia a la noción de clase textual de otros conceptos como, por ejemplo, el de tipo de texto o el de género discursivo. En esta línea, el tipo textual corresponde a una designación teórica de una forma específica de un texto, es decir, una categoría ligada a una teoría para la clasificación científica de textos que no necesariamente refleja el saber de la comunidad (Isenberg, 1987; Ciapuscio, 2003). Mientras que los géneros discursivos también son formas tipificadas de situaciones comunicativas, pero desde una mirada más amplia, desde una visión multidimensional corresponden a la concretización de una actividad sociodiscursiva. En consecuencia, los géneros son unidades altamente complejas y dinámicas, en cuya conformación son esenciales la dimensión cognitiva y la lingüística (Parodi y otros, 2015).

Dado que los miembros de la comunidad de nuestro interés son abogados y no especialistas en lengua, optamos por el concepto clase textual, lo que tiene dos ventajas esenciales. Primero, facilita el diálogo con los docentes entrevistados y, segundo, permite desarrollar una descripción y una propuesta didáctica con una terminología transparente, por lo que puede ser utilizada por un grupo amplio de interesados en el tema de la escritura en derecho.

\section{Metodología}

Este trabajo corresponde a una investigación cualitativa (Maxwell, 2012; Creswell y Creswell, 2017) con diseño fenomenológico (Bisquerra, 2009). Primero, porque no pretendemos generalizar de forma probabilística nuestros hallazgos y, segundo, porque nuestro propósito es reconstruir y describir la realidad como la observan los actores de un sistema social definido previamente (Creswell y Creswell, 2017), específicamente cómo la perciben los abogados que ejercen docencia en dos escuelas de derecho en Chile. En este marco, por una parte, planteamos como objetivo caracterizar las clases textuales de modalidad escrita producidas por estudiantes de derecho durante su formación en dos universidades chilenas y, por otra, presentar dos propuestas de enseñanza para trabajar dichas clases: una general (a nivel de familias de clases textuales) y una específica (a nivel de clase textual).

Las dos escuelas de derecho consideradas en la investigación tienen en común que pertenecen a universidades regionales que forman parte del Consejo de Rectores de las Universidades Chilenas. Esto implica que los estudiantes han pasado por un sistema de selección y admisión mediante un test estandarizado, que es la Prueba de 
Selección Universitaria. ${ }^{2}$ La diferencia entre las dos escuelas es que una pertenece a una institución estatal y la otra a una pública no estatal, según la distinción establecida por el Consejo de Rectores de las Universidades Chilenas. ${ }^{3}$ En total, entre ambas suman 64 docentes, quienes fueron invitados a participar en esta investigación.

Las técnicas de recolección de información utilizadas fueron un cuestionario en línea, un cuestionario en papel y una entrevista en profundidad. El cuestionario en línea se envió a los 64 docentes a través de un formulario Google. Luego, el cuestionario en papel se aplicó a los profesores que no respondieron en línea. Ambas versiones tenían las mismas preguntas: una primera parte con interrogantes de respuesta breve que recogían información personal y académica general; y una segunda parte con dos preguntas abiertas que apuntaban a ahondar en los textos producidos por los estudiantes en los cursos de cada profesor. Este cuestionario en papel se aplicó, además, a algunos profesores que habían contestado la versión en línea con información poco precisa. Así, esta versión del instrumento permitió aumentar el número de profesores consultados, profundizar en el conocimiento de la información entregada en línea, registrar nuevas clases de texto no señaladas en el cuestionario en línea y, finalmente, precisar información.

Una vez analizadas las respuestas obtenidas de los dos cuestionarios descritos, seleccionamos a algunos abogados-docentes para aplicar una entrevista selectiva en profundidad. La selección se hizo a través de un muestreo intencional, específicamente de tipo opinático (Bisquerra, 2009). El criterio de selección obedeció básicamente a la inclusión, por parte de los profesores, de actividades de producción de textos en los cursos que dictan. De esta manera, las entrevistas permitieron conocer con más detalle las clases textuales que son parte de la formación de los estudiantes de derecho y tanto sus características generales como lingüístico-discursivas. En la tabla 1, se muestra la cantidad de participantes en la investigación de acuerdo con los distintos métodos de recolección de datos.

Como se observa en la segunda fila de la tabla 1, de los 64 profesores que fueron invitados a contestar el cuestionario en línea o en papel, 50 de ellos respondieron $(78,13 \%)$. Por tanto, sobre la base de ese número están calculados los porcentajes de profesores participantes desglosados por tipo de institución. En los cuestionarios contamos con la colaboración de docentes con diferentes títulos profesionales (abogados, profesores, enfermeras), mientras que en las entrevistas en profundidad participaron exclusivamente abogados. Por otro lado, si bien el porcentaje de participantes en las entrevistas en profundidad es pequeño, se justifica por el tipo de investigación

2. «Historia del CRUCH», Consejo de Rectores de las Universidades chilenas, 2019, disponible en bit. $\mathrm{ly} / 2 \mathrm{~N}_{5} \mathrm{VzCC}$.

3. «Universidades CRUCH», Consejo de Rectores de las Universidades chilenas, 2019, disponible en bit.ly/30TTTV4. 
Tabla 1. Cantidad de participantes

\begin{tabular}{|lcc|}
\hline & Número & $\%$ \\
\hline Total de profesores invitados a contestar el cuestionario & 64 & $100 \%$ \\
Profesores que respondieron el cuestionario (muestra)* & 50 & $78,13 \%$ \\
Profesores que respondieron el cuestionario en la universidad estatal & 15 & $30 \%$ \\
Profesores que respondieron el cuestionario en la universidad no estatal & 35 & $70 \%$ \\
\hline Entrevista en profundidad a profesores de la institución estatal & 3 & $6 \%$ \\
\hline Entrevista en profundidad a profesores de la institución pública no estatal & 2 & $4 \%$ \\
\hline
\end{tabular}

Fuente: Elaboración propia

y por la metodología utilizada, como también por el alto grado de especialización de los participantes entrevistados.

Para analizar la información obtenida de la aplicación de los cuestionarios y de las entrevistas, utilizamos la técnica del análisis de contenido (Bautista, 2011). Ella, gracias a su carácter objetivo, sistemático y cualitativo (Krippendorff, 2019), permite la formulación de inferencias exhaustivas, reproducibles y válidas (Porta y Silva, 2003). Luego, a partir del listado de clases textuales identificadas, proponemos una clasificación para ellas, que fue levantada a partir de un enfoque ascendente-descendente en forma paralela (Tognini-Bonelli, 2001). En concreto, esto se hizo siguiendo los siguientes pasos: i) un análisis de la transcripción de las entrevistas; ii) una generación de categorías a partir de los contenidos presentes en el texto; iii) una construcción de grupos de categorías, considerando para ello afinidad temática; y iv) una conformación de propuestas a partir de las categorías agrupadas.

En síntesis, los procedimientos de investigación fueron: i) la recolección de datos sobre la base de encuestas y entrevistas; ii) la identificación de clases textuales a partir de un análisis de contenido; iii) la conformación de familias de clases textuales a través de un procedimiento inductivo; y iv) la generación de propuestas de enseñanza. En los dos últimos procedimientos contamos con la asesoría de un profesional que actuó como juez o par experto, específicamente, de un abogado que se desempeña como docente en carreras de Derecho de distintas universidades. Además, las dos propuestas didácticas formuladas fueron sometidas a un proceso de validación a través de una triangulación en la que participaron especialistas de distintas áreas: dos abogados, un estudiante de derecho y un profesor de habilidades comunicativas de la misma carrera. Luego, la propuesta específica a nivel de clase textual fue sometida a un nuevo proceso de validación, en el que participaron dos abogados-docentes y cuatro estudiantes de derecho. En consecuencia, se trata de propuestas validadas por distintos miembros de la comunidad a la que están dirigidas. 


\section{Análisis y discusión de resultados}

El primer resultado que ofrecemos en la tabla 2 corresponde al listado de clases textuales identificadas junto con su frecuencia absoluta de aparición $(\mathrm{N})$.

Es pertinente recordar que los datos recogidos en esta investigación se basan en lo referido por los docentes participantes, es decir, no existe un contraste con otro tipo de información como, por ejemplo, planificaciones de clases, evaluaciones, entrevistas a los estudiantes, etcétera. Además, se debe tener presente que este trabajo es sobre las clases textuales identificadas a partir del discurso de los abogados, lo que implica que hemos mantenido los mismos términos que ellos utilizaron al encuestarlos y, por tanto, las categorías levantadas no han sido agrupadas. Esto explica que, por ejemplo, en la tabla 2 haya más de un nombre para lo que podría corresponder a un mismo género, como ocurre en el caso de «prueba» $y$ «prueba escrita» $\mathrm{o}$ «informe» $\mathrm{e}$ «informe escrito». También esclarece el hecho de que haya términos que no necesariamente podrían equipararse con un género, sino más bien con un modo de organización discursiva («textos narrativos») o con otros conceptos o niveles («discursivo»).

Tabla 2. Clases textuales mencionadas por los docentes encuestados

\begin{tabular}{|llll|}
\hline \multicolumn{2}{c}{ Escuela de Derecho (Universidad estatal) } & \multicolumn{2}{c}{ Escuela de Derecho (Universidad no estatal) } \\
\cline { 2 - 3 } & Núm. & Clase & Núm. \\
\hline Análisis de jurisprudencia & 1 & Análisis de jurisprudencia & 3 \\
\hline Análisis de sentencia & 1 & Análisis de sentencia & 1 \\
Artículo de investigación & 1 & $\begin{array}{l}\text { Análisis de una sentencia de inaplicabilidad del tribunal } \\
\text { constitucional }\end{array}$ & 1 \\
\hline Comentario crítico & 1 & Avance de tesis & 1 \\
\hline Contratos & 3 & Carta de aviso de término de contrato & 1 \\
\hline Demanda & 2 & Comentarios de jurisprudencia & 2 \\
\hline Discursivo & 1 & Contrato de trabajo & 1 \\
\hline Ensayo & 2 & Contratos & 1 \\
\hline Ensayos acerca de temas analizados en clase & 1 & Control de lectura & 1 \\
\hline Escritos y presentaciones para tribunales de justicia & 1 & Discurso argumentativo & 1 \\
\hline Escritura pública & 1 & Elaboración de recursos de nulidad & 1 \\
\hline Informe de investigación & 1 & Elaboración de recursos de unificación de jurisprudenciaa & 1 \\
\hline Informe en Derecho & 1 & Ensayo & 4 \\
\hline Informes & 2 & Ensayo argumentativo & 1 \\
\hline Informes de estado de avance de causas & 1 & Ensayo en inglés & 1 \\
\hline Informes de sentencias relevantes & 1 & Ensayo modificado & 1 \\
\hline Informes en Derecho sobre temas jurídicos & 1 & Escrito judicial & 1 \\
\hline Mandatos & 1 & Escritos de contestación de demanda & 1 \\
\hline
\end{tabular}




\begin{tabular}{|c|c|c|c|}
\hline Monografía & 1 & Escritos jurídicos & 1 \\
\hline Pruebas & 2 & Escritura pública & 1 \\
\hline Pruebas escritas & 5 & Evaluación escrita & 22 \\
\hline Reporte & 1 & Evaluación escrita solemne & 1 \\
\hline Seminario de memoria & 1 & Guión de un debate & 1 \\
\hline Sentencia & 1 & Informe en Derecho & 2 \\
\hline Solución de casos & 1 & Informe en Derecho modificado & 1 \\
\hline Tramitación de la demanda & 1 & Informe escrito & 1 \\
\hline \multirow[t]{19}{*}{ Tramitaciones judiciales } & 1 & Informes & 2 \\
\hline & & Informes de análisis de casos & 1 \\
\hline & & Oficio & 1 \\
\hline & & $\begin{array}{l}\text { Portafolio conformado por actividades en clases por medio } \\
\text { del cual determinan problemas jurídicos }\end{array}$ & 2 \\
\hline & & Portafolio de análisis de casos & 1 \\
\hline & & $\begin{array}{l}\text { Portafolio que reúne trabajos en base a lecturas dadas en } \\
\text { clases }\end{array}$ & 2 \\
\hline & & Prueba escrita & 1 \\
\hline & & $\begin{array}{l}\text { Pruebas con ítem de aplicación de resolución de casos } \\
\text { reales }\end{array}$ & 1 \\
\hline & & $\begin{array}{l}\text { Pruebas escritas: alternativas/verdadero-falso/ resolución } \\
\text { de un caso }\end{array}$ & 1 \\
\hline & & Redacción de demandas & 1 \\
\hline & & Reglamento & 1 \\
\hline & & Requerimiento de inaplicabilidad & 1 \\
\hline & & Resolución & 1 \\
\hline & & Respuestas de desarrollo en pruebas de la asignatura & 1 \\
\hline & & Textos jurídicos & 1 \\
\hline & & Textos narrativos & 1 \\
\hline & & Trabajo de análisis (contenido por definir) & 1 \\
\hline & & Trabajos de investigación & 3 \\
\hline & & $\begin{array}{l}\text { Trabajos escritos: teoría fáctica del caso, teoría jurídica, } \\
\text { teoría probatoria }\end{array}$ & 1 \\
\hline Total & 37 & Total & 78 \\
\hline
\end{tabular}

En cuanto a la clase más frecuente, en la institución estatal corresponde a la «prueba escrita» ( 5 menciones), mientras que en la institución pública no estatal es la «evaluación escrita» (22 alusiones). En consecuencia, en ambas escuelas de derecho la instancia en la que más escriben los estudiantes es en pruebas, lo que implica que prevalecen las actividades de escritura asociadas a un género académico tradicional más que a uno propio de la disciplina. Creemos que la predominancia de la prueba escrita podría deberse, primero, a que se trata de un tipo de evaluación omnipresen- 
te, de larga data y utilizado internacionalmente (Villarroel y otros, 2018). Segundo, como indican investigaciones recientes, porque los docentes en general muestran resistencia para cambiar la evaluación formal a través de pruebas y exámenes escritos, sosteniendo la creencia de que aprender involucra la reproducción literal del saber, la acumulación de datos, conceptos y habilidades básicas (Villarroel y otros, 2019). Esto podría adquirir aún más relevancia en una carrera tradicional como Derecho, en la que el papel de la memoria y la acumulación de conocimientos ha tenido tradicionalmente un rol preponderante. Además, se ha destacado de las pruebas escritas el hecho de que permiten corroborar el aprendizaje que los estudiantes han logrado interiorizar, asimilar, comprender y construir, de modo que constituyen una oportunidad para verificar saberes y, al mismo tiempo, probar la calidad del trabajo docente (Herrera, 2015). A pesar de lo anterior, no desconocemos las críticas a este tipo de evaluación (Riquelme y otros, 2017).

Otro dato interesante es que, en las dos escuelas de derecho, la gran mayoría de los docentes declara que sí realiza actividades de escritura con sus estudiantes, específicamente un 93,33\% en la institución estatal y un 97,14\% en la institución pública no estatal (véase el anexo 1). Si bien las frecuencias relativas son distintas en términos de frecuencia absoluta, en ambas escuelas solo un docente no realiza actividades de escritura y, curiosamente, corresponde a la misma persona. Por tanto, podríamos atribuir la ausencia de actividades de escritura a un estilo de enseñanza personal más que a una política de las escuelas. En esta línea, la variedad de clases textuales declaradas por cada docente varía entre o y 8 en la institución estatal y entre o y 13 en la institución pública no estatal. En consecuencia, ambas escuelas cuentan con docentes que no someten a sus estudiantes a la producción de ningún texto, mientras que hay otros que los llevan a producir una gran variedad de ellos. Esto podría incidir en la diversidad de textos que domina un estudiante al finalizar su formación de pregrado. Por ejemplo, un egresado podría dominar la producción de una variedad importante de textos de derecho del trabajo, pero ninguno de derecho de aguas.

También es interesante la relación que podría existir entre la formación profesional de los docentes y las clases textuales que desarrollan con sus estudiantes (véase el anexo 2). Así, si bien en ambas escuelas predominan ampliamente los abogados por sobre cualquier otro tipo de profesional, la proporción es un poco mayor en la institución pública no estatal $(88,57 \%)$ por sobre la institución estatal (80\%). Esto indica que los estudiantes de las escuelas investigadas no solo son formados por abogados, sino también por profesores de inglés y castellano, enfermeras e ingenieros. Este resultado aporta información que otras investigaciones desconocen toda vez que afirman que la comunidad de profesores de derecho está constituida por abogados, unos que se desempeñan como académicos a tiempo completo y otros dedicados principalmente al ejercicio de su profesión, y adicionalmente se desempeñan como docentes (Muñoz, 2014; Aguilar, 2017). 
En este orden de ideas, advertimos que la institución pública no estatal tiene una mayor variedad de profesionales, lo que incide en la variedad de textos que producen los futuros abogados. Por ejemplo, los estudiantes de la institución pública no estatal, además de escribir textos académicos generales y otros propios de su formación, producen textos narrativos y ensayos en inglés con la profesora de esta asignatura; guiones de debates, ensayos y discursos argumentativos con la profesora de Castellano y textos sobre bioética con la enfermera. Los estudiantes de la institución estatal, en cambio, privilegiarían los textos especializados a través de la producción de una solución de casos empresariales con una ingeniera, un informe en el que demuestran sus habilidades informáticas con otro ingeniero y un artículo de investigación con la profesora de Castellano. En síntesis, la formación de los docentes que integran el cuerpo académico de cada escuela influye en los tipos y variedad de textos que desarrollan los abogados en formación y, en consecuencia, marca una diferencia en la preparación de los estudiantes. Así, los estudiantes de la institución pública no estatal, a partir de los docentes no abogados, tendrían una formación más fuerte en la producción de textos que los conduce a desarrollar sus habilidades comunicativas y de bioética, mientras que los estudiantes de la institución estatal fortalecerían sus habilidades empresariales, informáticas y de comunicación científica.

Un aspecto que llama la atención de las clases textuales mencionadas por los profesores entrevistados es que no aparecen algunos textos propios de la formación de estudiantes de derecho, como los incidentes y querellas, entre otros. Este hecho resulta llamativo, sobre todo si consideramos que en la investigación contamos con las respuestas de más del 75\% de los profesores de las escuelas estudiadas. Por supuesto, cabe la posibilidad de que los profesores que no participaron en la investigación aborden los textos señalados o que los profesores encuestados no los hayan mencionado.

A partir de estos resultados descriptivos proponemos una clasificación de las clases textuales identificadas a partir de la determinación del ámbito al que pertenecen. Para ello, como adelantamos en la metodología, realizamos un análisis inductivo o bottom-up, lo que implica que no utilizamos clasificaciones preexistentes, sino que nuestra propuesta proviene del análisis de los datos. Nuestra clasificación se presenta en la figura 1.

Como se puede advertir en la figura 1, todas las clases textuales identificadas en esta investigación y presentadas en la tabla 2 se han agrupado en cuatro familias: académicas transversales, académicas transversales adaptadas, de transición y profesionales. Todas aparecen caracterizadas en los rectángulos en blanco de la figura 1 y, además, se ejemplifican con las clases textuales específicas en los recuadros grises. Esta propuesta de clasificación y la incorporación de cada clase en una familia fue consultada y validada por un abogado que ejerce la docencia a estudiantes de derecho en distintos niveles. Un aspecto interesante de estas familias es que existe un nivel de progresión de menor a mayor especialización, representado por las flechas en el 


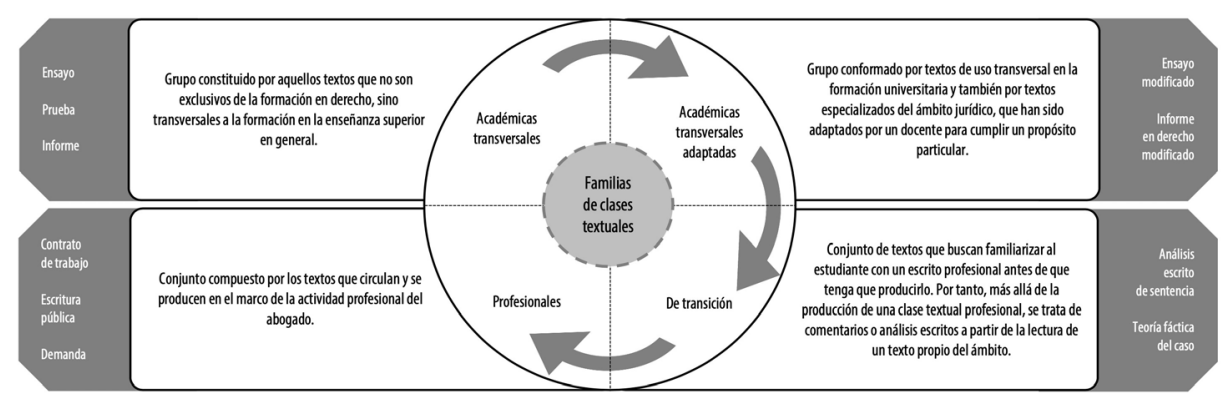

Figura 1. Propuesta de clasificación de las clases textuales identificadas.

centro del diagrama, desde las clases académicas transversales hasta las profesionales. Por ejemplo, los estudiantes de niveles iniciales producen, casi exclusivamente, clases textuales académicas transversales, mientras que las clases profesionales están reservadas para estudiantes de cursos superiores o cercanos al egreso, ya que es en esta etapa final de la formación en la que se producen los textos propios de la profesión de un abogado.

Desde nuestro punto de vista, las clases académicas transversales adaptadas y las de transición resultan muy interesantes. En primer lugar, porque no han sido descritas en la bibliografía revisada. En segundo lugar, porque evidencian el carácter de proceso de la escritura disciplinar y la necesidad de formar a los estudiantes en la cultura de su disciplina (Hyland, 2013). Y finalmente, en tercer lugar, porque implican un proceso didáctico creativo por parte del docente. Así, en las clases académicas transversales adaptadas, el profesor adecúa una clase textual transversal para cumplir un propósito particular y acercar al estudiante al conocimiento disciplinar. Por ejemplo, en el caso del ensayo modificado, el docente hizo ajustes a la clase transversal ensayo para que sus estudiantes desarrollaran su escrito acorde con las normas de un coloquio sobre un tema jurídico específico en el que participaron. Por otro lado, en el caso de las clases de transición, el profesor acerca al estudiante a los textos profesionales a partir de un análisis escrito. Ejemplo de ello es el comentario escrito de una sentencia de inaplicabilidad del Tribunal Constitucional, actividad que prepara al estudiante para que pueda escribir su propio requerimiento de inaplicabilidad.

En la figura 2, presentamos la frecuencia relativa de las familias de clases textuales que constituyen nuestra propuesta.

Como podemos observar, en ambas escuelas de derecho, las clases textuales menos frecuentes son las que agrupamos bajo el nombre de transversales adaptadas, lo que resulta esperable, ya que son ejercicios específicos de escritura que algún profesor ha creado para una actividad concreta. Otro dato interesante es el hecho de que existe una diferencia entre las escuelas en la familia de clases textuales más utilizada. Así, en la institución estatal lo más recurrente son las clases transversales, lo que sugiere 


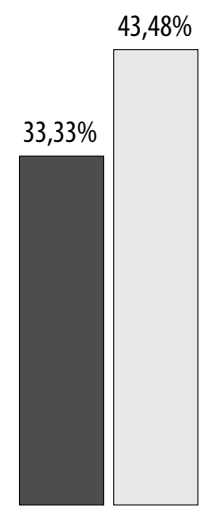

Transversal

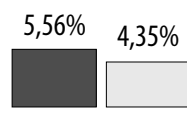

Transversal adaptada

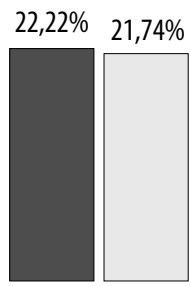

Transición

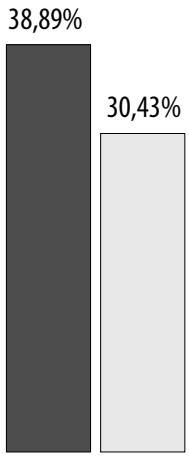

Profesional

Figura 2. Frecuencia relativa de las familias de clases textuales propuestas.

un mayor interés en la formación general de sus estudiantes. Esto podría atribuirse al hecho de que es una escuela de derecho relativamente nueva, por lo que, quizás, el foco aún no está sobre el desempeño profesional de los estudiantes. En la institución pública no estatal, en cambio, lo más recurrente son las clases profesionales, lo que apuntaría a un énfasis en la preparación de los estudiantes para su desempeño profesional. De hecho, a diferencia de la institución estatal, los estudiantes de la institución pública no estatal, desde su segundo año de carrera, ya redactan una clase textual profesional, es decir, un requerimiento de inaplicabilidad. Otra explicación plausible podría ser la fuerte presencia en la institución pública no estatal de una Clínica Jurídica, por lo que los estudiantes tendrían mayor posibilidad de desarrollar textos propios de su profesión.

Una vez identificadas, analizadas y agrupadas las diferentes clases textuales, desarrollamos una propuesta para la enseñanza de las distintas familias de clases textuales. Ella se basa en los datos recogidos, la clasificación propuesta de familias, la asesoría técnica recibida y nuestra experticia como docentes o estudiantes de derecho. Esta propuesta didáctica posee dos partes que corresponden a dos niveles de especificidad. Primero, en un nivel más general, presentamos una propuesta a partir de las familias de clases descritas anteriormente y, segundo, en un nivel más específico, desarrollamos una propuesta al nivel de cada clase textual.

A continuación, detallamos la propuesta a nivel de familias de clases. Para ello presentamos cuatro figuras, una por cada familia, en las que ofrecemos una serie de criterios a partir de los que se puede abordar la producción de los textos asociados a cada familia. Dichos criterios son siete, cada uno de los cuales relacionamos con una pregunta con el fin de facilitar tanto su caracterización como el trabajo didáctico: 
a) Elaboración de párrafos en torno a distintos temas, con variados propósitos, diferentes formas de organización, utilizando diversos mecanismos de cohesión y asgurando la existencia de progresión temática.

b) Producción de distintas clases de textos de la familia (ensayos, informes, resúmenes), generados a partir de lecturas previas en torno a una temática.

c) Generación de clases de textos especíicos (ensayos, informes, resúmenes) a partir de la revisión grupal 0 individual de los modelos textuales y sus rasgos lingüístico-discursivos.

d) Desarrollo de cedularios en los que se práctica cómo responder las evaluaciones escritas.

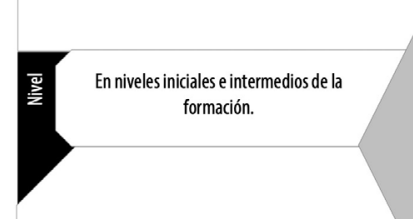

Integrar estrategias de retroalimentación y reforzamiento de las habilidades transversales como también del conocimiento disciplinar de base, transformándose, por tanto, en un mediador en la construcción de los aprendizajes a partir de la elaboración del texto.

\section{Propósito}

Desarrollary fortalecer habilidades trasnversales para enfrentar la vida universitaria 0 adquirir conocimientos específicos de base en torno al área de formación a través de la utilización de dases textuales relativamente sencillas.

Evaluaciones con intencionalidad formativa rientaciones para la mejora, otras, asociadas una calificación, a partir del desempeño alcanzado por el estudiante frente a una tarea.
Ensayos, informes, pruebas, entre otros.

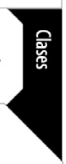

Participar activamente en las actividades de aprendizaje propuestas por el docente, siendo capaz de elaborar textos en los que se ponga en juego el uso de habilidades transversales, como también el conocimiento disciplinar de base que ha adquirido. Todo esto, considerando las mejoras necesarias a partir del proceso de retroalimentación realizado por el docente.

Figura 3. Familia de clases académicas transversales

a) Propósito: ¿Cuál es el propósito de que los estudiantes produzcan textos de las clases pertenecientes a esta familia?

b) Nivel: ¿En qué nivel, principalmente, los estudiantes se enfrentan a las clases textuales que constituyen esta familia?

c) Clases: ¿Qué clases textuales forman parte de esta familia?

d) Rol del docente: ¿Cuál es el rol que cumple el docente al integrar en el proceso formativo las clases de textos que constituyen esta familia?

e) Rol del estudiante: ¿Qué rol cumple el estudiante al enfrentarse a las clases que conforman esta familia?

f) Actividades de aprendizaje: ¿Qué tipos de actividades de aprendizaje son factibles de implementar para la escritura de las clases de textos pertenecientes a esta familia?

g) Estrategias de evaluación: ¿Qué estrategias de evaluación son más adecuadas para evaluar actividades de aprendizaje asociadas a la producción de las clases textuales pertenecientes a esta familia?

En la figura 3 ofrecemos la propuesta para las clases académicas transversales.

En la figura 4 graficamos la propuesta para las clases académicas transversales adaptadas.

A continuación, en la figura 5 organizamos los elementos de la propuesta para las clases de transición. 
a) Producción de clases de textos con propósitos y formas de organización específicas, según los requerimientos de la tarea.

b) Revisión de clases de textos modificados para efectos de reconocer sus características lingüístico-discursivas y seguir determinados patrones en la producción escrita propia.

c) Revisión conjunta con compañeros, o con el docente, de clases textuales que permitan profundizar y aclarar una temática disciplinar compleja.

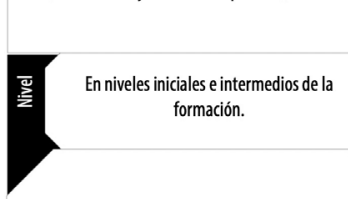

Implementar estrategias de modelamiento cognitivo $y$ acompañamiento en el proceso de construcción de variadas clases de textos, asi como también integrar estrategias explicativas que posibiliten la comprensión del conocimiento disciplinar complejo.

\section{Propósito}

Iniciar un proceso de acercamiento al sabe disciplinar de manera sencilla, asi como también conocer los rasgos lingüístico-discursivos centrales de las clases de esta familia, a fin de desarrollar habilidades que permitan dar respuesta a las tareas de escritura propias de ámbito académico.

Evaluaciones con intencionalidades formativa y sumativa que impliquen dar cuenta de la adquisición de un conocimiento disciplinar complejo a través de la producción de una clase de texto que ha sido abordada.
Ensayo modificado, informe en derecho modificado, entre otros.

Responder a cada una de las tareas propuestas que buscan, a través de la escritura, acercar el conocimiento disciplinar complejo y construir variadas clases de textos de carácter académico, respetando las modificaciones introducidas por el docente a la clase textual correspondiente.

Rol del estudiante

Figura 4. Familia de clases académicas transversales adaptadas

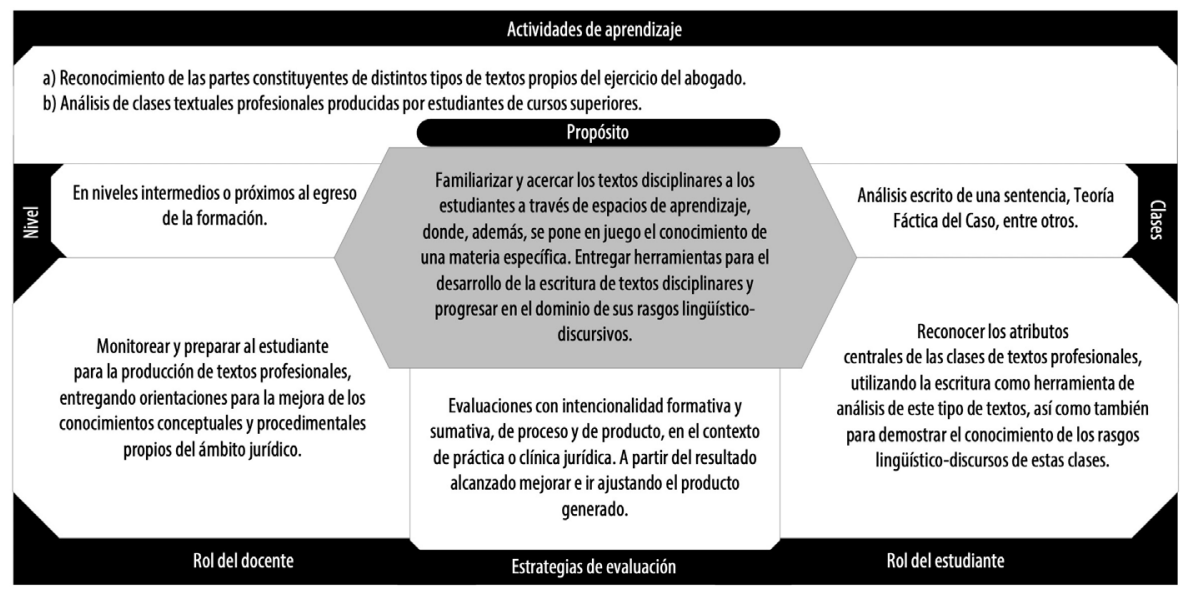

Figura 5. Familia de clases de transición.

Por último, en la figura 6 ofrecemos los elementos de la propuesta para las clases textuales profesionales.

En síntesis, en esta primera propuesta presentamos siete criterios que pueden ayudar al docente o al estudiante en el proceso de producción de las clases textuales que componen las cuatro familias que hemos formulado. En el caso del estudiante, le permite conocer las características de los grupos de texto que debe producir. En el caso del docente, brinda mayor cantidad de herramientas para enseñar los textos asociados a las cuatro familias identificadas.

Si bien esta primera propuesta constituye un avance concreto en la didáctica de la escritura en derecho, las indicaciones se presentan a nivel de familias de clases 


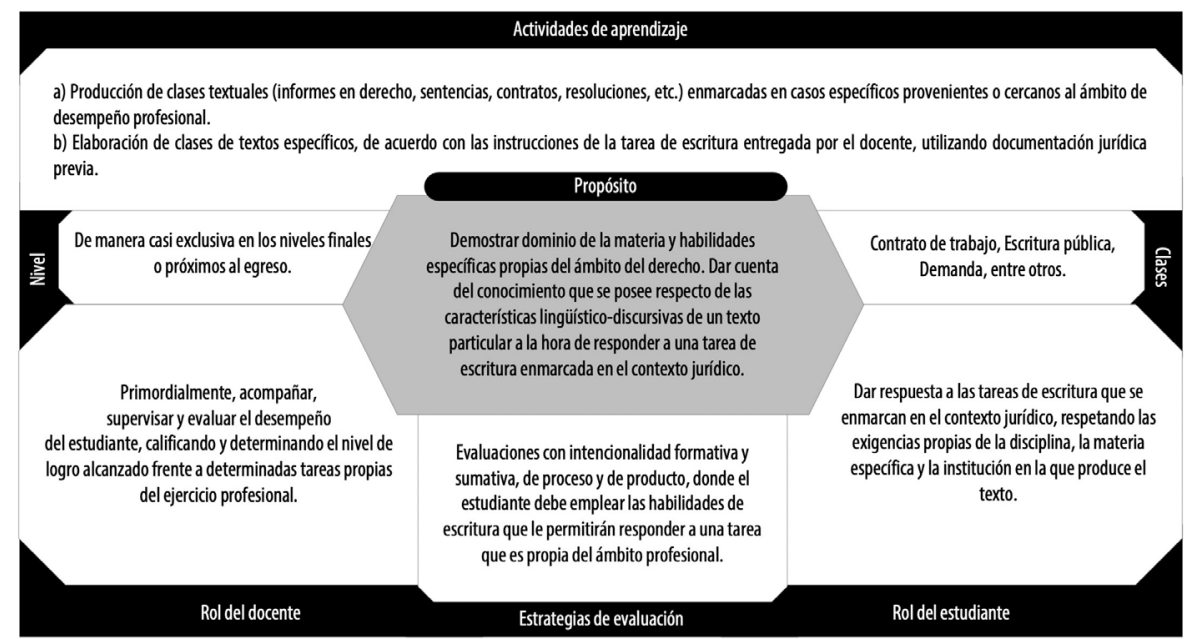

Figura 6. Familia de clases profesionales.

textuales y no de textos específicos. Por esto, la segunda parte de nuestra propuesta contempla la caracterización de una clase textual específica. Esta descripción permite entregar algunos lineamientos para abordar el proceso de enseñanza de la escritura de un texto disciplinar en este ámbito. A nuestro juicio, algunos de los criterios pertinentes para la caracterización de cada clase textual son: familia a la que pertenece el texto (según la clasificación propuesta en esta investigación), definición general del texto, propósito comunicativo, estructura textual u organización de la información, ámbito de circulación, entre otros.

La especificación y concretización conjunta de todos los criterios mencionados, por un lado, permitirá al estudiante crear sus propios textos disciplinares y así evitar recurrir a la adaptación de formatos preestablecidos, como es común en la formación de estudiantes de derecho. Por otro lado, brindará al docente la posibilidad de guiar con mayor facilidad a los abogados en formación en la producción de textos propios de su disciplina. Esta propuesta está constituida por tres partes. En primer lugar, por una caracterización general en la que se presenta la situación retórica general del texto que se debe producir, es decir, identificación de la clase textual, familia a la que pertenece, propósito comunicativo, audiencia, ámbito de circulación y sujeto productor. Por ejemplo, para redactar una sentencia civil, que es una de las clases textuales mencionadas por los entrevistados, la caracterización general es la que se presenta en la figura 7 , que constituye la primera parte de nuestra propuesta a nivel textual. Cabe señalar que para ejemplificar esta parte de nuestra propuesta hemos elegido un tipo específico de sentencia civil, que es la de primera instancia. 


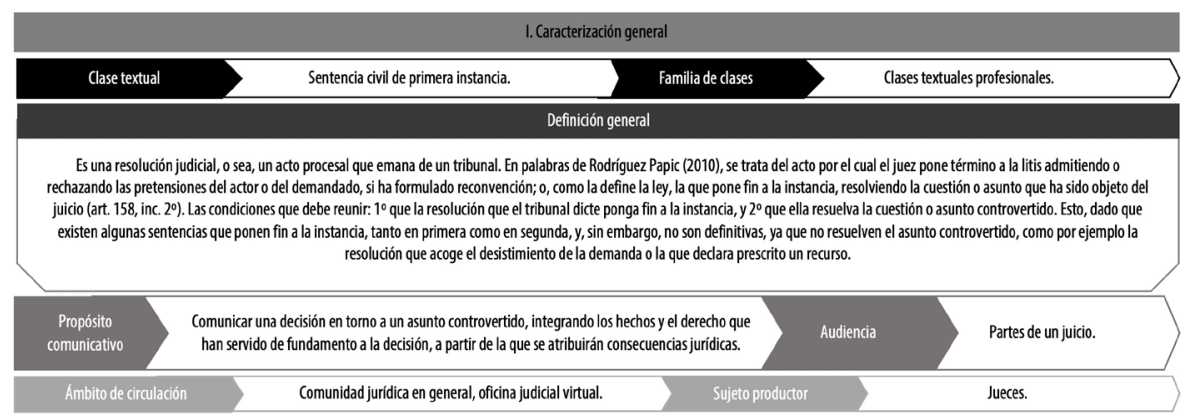

Figura 7. Primera parte de la propuesta didáctica específica «caracterización general».

La función de cada uno de los criterios definidos en esta primera parte de la propuesta es la siguiente:

a) Familia de clases: Permite situar al estudiante en la familia general a la que pertenece el texto que debe producir.

b) Definición general: Ayuda a figurar un modelo general de la tarea de escritura que debe desarrollarse.

c) Propósito comunicativo: A partir de este tercer criterio, el usuario puede tener claridad sobre el objetivo del texto que se debe redactar, considerando que «los textos, como instancias concretas de una lengua particular, son producidos por los individuos en forma intencionada» (Parodi, Ibáñez y Venegas, 2009: 83).

d) Audiencia: Apunta al destinatario final del texto, es decir, no al profesor como destinatario y evaluador de la tarea encomendada al estudiante, sino que da cuenta de la audiencia figurada del texto profesional que se produce, por ejemplo, un juez, otro abogado, etcétera.

e) Ámbito de circulación: Alude al contexto o espacio profesional del abogado en que el texto es utilizado (tribunales, por ejemplo). Esto permitirá al abogado en formación conocer la naturaleza de su escrito. Así, por ejemplo, al saber que el texto que debe producir circula en tribunales, sabrá que se le ha solicitado un texto de práctica forense.

f) Sujeto productor: Indica el rol que cumple quien escribe el texto, entendiendo que el estudiante debe figurar un escritor experto, en un rol jurídico específico (fiscal, abogado, etcétera). De esta forma, el estudiante aprenderá a diferenciar entre los escritos que presenta un fiscal de aquellos a través de los cuales comunica el juez, como son las sentencias o las resoluciones. La explicitación del rol o, más específicamente, de quién actuará como autor del texto (abogado, juez, etcétera), es relevante porque existen casos en que, por ejemplo, un abogado redacta una sentencia, pero lo hace para adelantar el trabajo del juez, quien revisará el texto y lo hará propio, ya que, en definitiva, él es el responsable de emitir una sentencia. 


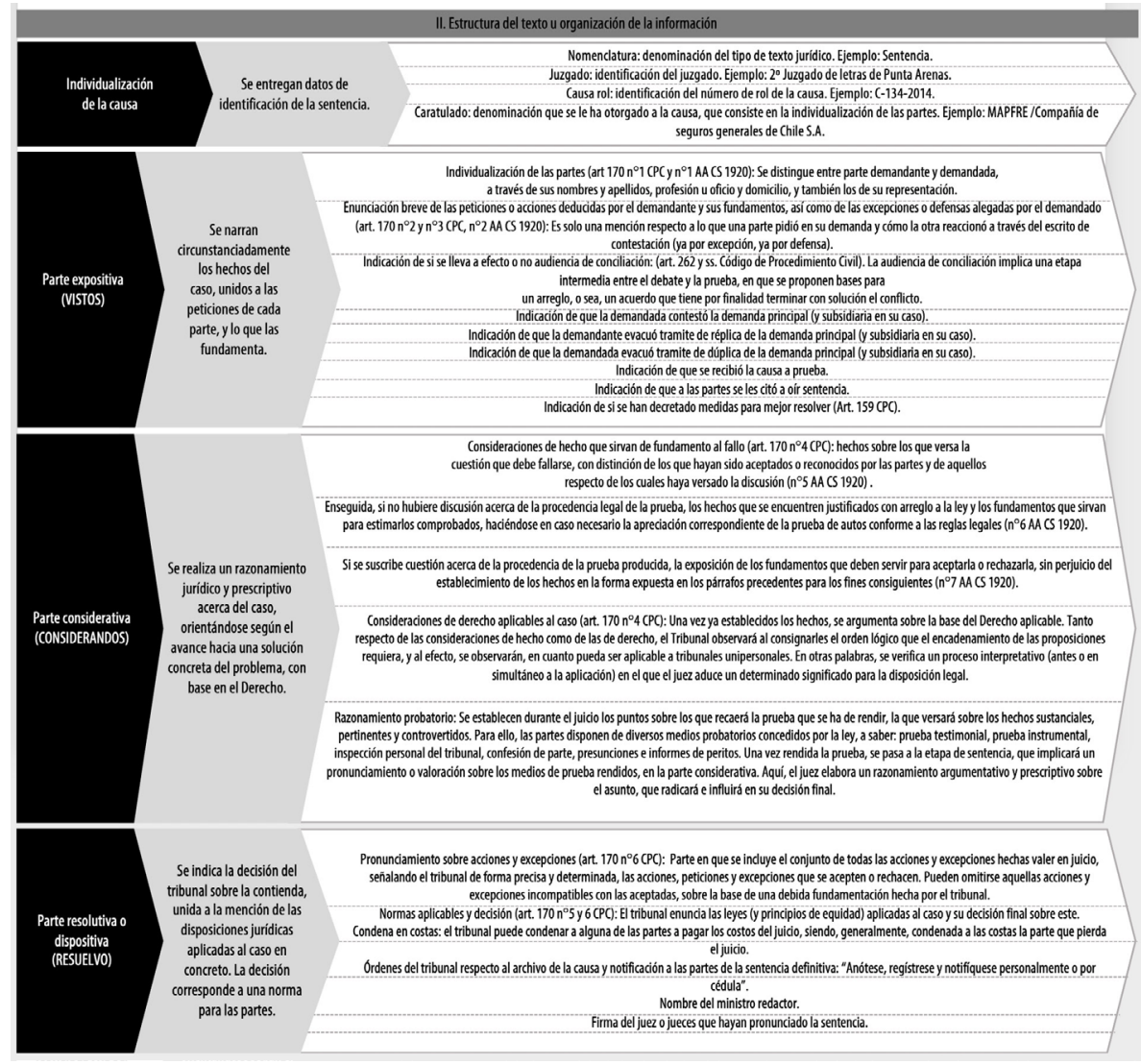

Figura 8. Segunda parte de la propuesta didáctica específica «estructura del texto u organización de la información».

Una vez que el productor del texto se ha formado una idea general de la tarea de escritura, podría pasar a la segunda parte de la propuesta, que corresponde a la descripción de la estructura del texto u organización de la información. Esta caracterización permitirá al abogado en formación conocer o recordar la estructura específica del texto en cuestión, sus partes y cómo organizar la información dentro de esa estructura. Por ejemplo, siguiendo con el caso de la sentencia civil de primera instancia, el estudiante sabrá cuáles son las partes concretas que debe considerar para estructurar su texto (individualización de la causa, parte expositiva, parte considerativa, parte resolutiva o dispositiva), una breve definición de cada una de ellas y los elementos específicos que debe incluir en cada parte junto con una breve caracterización. Además, varios de estos elementos incluyen la referencia de códigos o artículos que el estudiante podría revisar para profundizar la información sobre algún elemento. Esta segunda parte de la propuesta se presenta en la figura 8. 


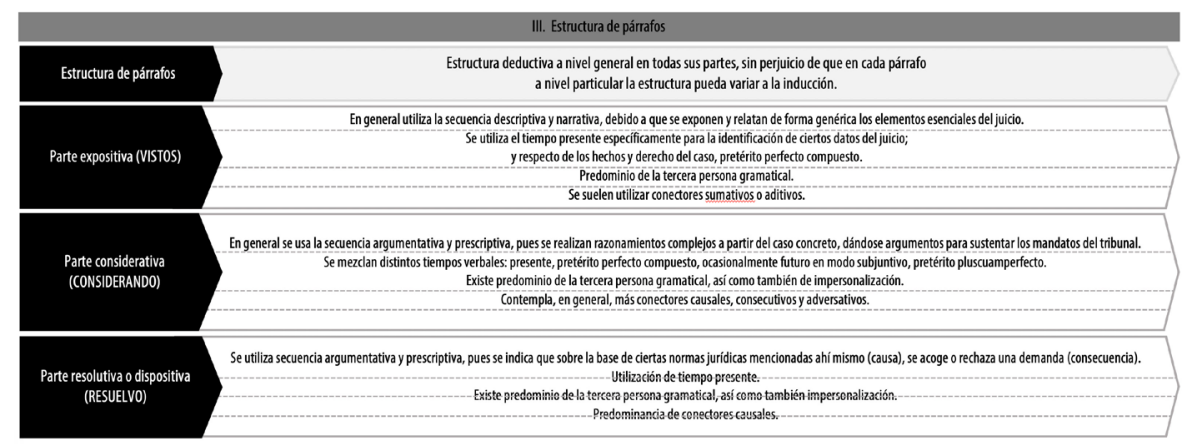

Figura 9. Tercera parte de la propuesta didáctica específica «estructura de párrafos».

Por último, la tercera parte de nuestra propuesta corresponde a la estructura de párrafos, que permite que el estudiante conozca las características lingüístico-discursivas de cada parte de del texto que debe producir (tiempos verbales, marcadores discursivos predominantes, secuencias textuales prototípicas, etcétera). Entonces, con esta información, el productor del texto sabrá cómo completar la estructura indicada en la segunda parte de nuestra propuesta. A continuación, la parte correspondiente a la estructura de párrafos se presenta en la figura 9.

La suma de las tres partes de nuestra propuesta permitirá a los estudiantes de derecho conocer cabalmente la clase textual que deben producir y, además, tener algunas orientaciones, por ejemplo, sobre el registro, el nivel de especialización del léxico, el conocimiento compartido, las características lingüístico-discursivas, etcétera. Si consideramos que la formación profesional es un proceso paulatino a través del cual se van produciendo cambios en el lenguaje del sujeto en formación (Farfán, 2019), nuestra propuesta será un gran aporte en este sentido, ya que entrega herramientas concretas para apoyar el proceso de formación e inserción disciplinar de los futuros abogados. Así, por ejemplo, para trabajar los distintos tipos de clases textuales, el profesor puede entregar la matriz completa al estudiante (figuras 7,8 y 9) o, incluso, en un nivel más avanzado, el estudiante podría recibir la matriz con las descripciones en blanco e iniciar su trabajo completando la información correspondiente a cada criterio. Además, la propuesta podría utilizarse como herramienta de evaluación para determinar el nivel de conocimiento que posee un estudiante sobre algún texto utilizado en su formación profesional.

Esta propuesta específica presentada fue sometida a juicio de pares con el fin de evaluar si los criterios considerados eran pertinentes y si las descripciones de cada uno de ellos eran adecuadas. Concretamente, participaron dos abogados que, además de ejercer su profesión, se desempeñan como docentes en distintas escuelas de derecho, y cuatro estudiantes de cuarto año. Ellos evaluaron la matriz sobre la base de los siguientes criterios: aspectos positivos de la propuesta, aspectos mejorables, 
aspectos no considerados y observaciones generales. Los comentarios fueron analizados e incorporados en la versión final de la propuesta. Uno de los aspectos positivos que destaca uno de los abogados es que "podría ser útil para que los alumnos se familiaricen con la sentencia civil, sus requisitos y, de esta forma, puedan estudiarlas y comprenderlas de mejor manera». Por otra parte, el otro abogado menciona que «ofrece un claro análisis de las partes que componen una sentencia, identificando y distinguiendo partes, pero, además, estableciendo vinculaciones entre unas y otras. Es interesante que la propuesta haga referencia a la «estructura de los párrafos».

Por su parte, uno de los estudiantes evaluadores subraya que:

Las matrices resuelven muchas de las inquietudes de la materia que pudieran tener tanto un extraño al área del derecho como los estudiantes y abogados. Además, el texto es muy explicativo de las funciones específicas y de las finalidades comunicativas del procedimiento civil, lo que permite darles un enfoque práctico asociado a conocimientos previos de dominio más o menos especializado, facilitando la comprensión y generando aprendizaje.

Por su parte, otro de los estudiantes menciona lo siguiente: «Me parece útil, ya que se enfoca a todo público que desee aprender de las materias de que se trata [...] Se puede utilizar pedagógicamente». Adicionalmente, otro de los estudiantes evaluadores afirma que:

La terminología utilizada es simple y básica, por lo que permite que las personas que no tienen cercanía con el derecho o que están en los primeros años de la carrera puedan enfrentar de mejor manera la lectura como redacción de una sentencia, sobre todo considerando la complejidad muchas veces de ciertas instituciones o de las terminologías que se usan en el área [...]. La utilización de colores, esquemas y la distribución de la información es óptima para entender la estructura de una sentencia.

Finalmente, el último de los estudiantes menciona que:

Es destacable lo claro que resulta para el lector encontrar los aspectos esenciales de una sentencia, sobre todo considerando que la propuesta se enmarca en un contexto de enseñanza del derecho, donde muchas veces resulta difícil para el estudiante trabajar y elaborar una sentencia por la complejidad de la estructura y del lenguaje, por ello estimo que con esta matriz se puede obtener un acercamiento más ameno y claro para el estudiante. Lo anteriormente dicho se realza si consideramos que el alumno que tendrá a su disposición esta matriz es un estudiante de primer o segundo año, ya que el estadio de formación que suponen esos grados hace que sea difícil para el alumno elaborar una sentencia por sí mismo, sin una estructura (como la de la propuesta) que sirva como guía. 
En cuanto a los aspectos mejorables o no considerados indicados por los evaluadores, fueron revisados y se incluyeron en la propuesta final presentada. En síntesis, profesores y estudiantes de derecho tienen una valoración positiva de la propuesta que presentamos.

\section{Conclusión}

Para concluir, es posible afirmar que hemos consumado los dos objetivos propuestos en esta investigación. Por una parte, hemos identificado, a partir del discurso de los docentes, las clases textuales producidas por los estudiantes durante su formación. Así, descubrimos que la clase más frecuente es la prueba escrita, predominancia que atribuimos, entre otras razones, a que se trata de un tipo de evaluación omnipresente, de larga data y utilizado internacionalmente (Villarroel y otros, 2018), independientemente de la disciplina. Por otra parte, para caracterizar las clases identificadas, sobre la base de los datos proponemos la clasificación de las clases textuales en familias. Con base en estos resultados, y en atención al segundo objetivo de este trabajo, presentamos dos propuestas didácticas para abordar las diversas clases textuales que se utilizan en la formación en derecho.

Creemos que la investigación presentada constituye un avance para superar algunos de los problemas persistentes en otros trabajos desarrollados en el ámbito de la escritura jurídica. Así, en este trabajo, la identificación de las clases textuales se ha hecho sobre la base de lo señalado por los miembros de la comunidad disciplinar, por lo que no seleccionamos arbitrariamente los textos aquí incluidos.

Los valores de nuestra propuesta que, por cierto, la diferencian de los trabajos existentes hasta la fecha, radican en cuatro puntos centrales. Primero, no solo clasificamos textos como hacen otras investigaciones (Aguilar, 2017), sino que además avanzamos hacia una propuesta didáctica para enseñar la producción de los textos que, según los profesores entrevistados, deben desarrollar los estudiantes de derecho a lo largo de su formación. Segundo, destacamos el carácter interdisciplinario de nuestra propuesta elaborada por especialistas de distintas áreas: lingüística (concretamente, escritura académica y científica), educación, y derecho. En este sentido, hemos considerado a los distintos actores involucrados en el proceso de formación de la producción de textos disciplinares de derecho. Esto contribuye a superar la mirada restringida sobre el tema, es decir, exclusivamente lingüística o disciplinar. En tercer lugar, y en línea con nuestra preocupación didáctica, para evitar el problema de la utilización de conceptos que pueden resultar complejos y confusos, como ocurre con el de género discursivo, empleamos la noción de clase textual. Ella permite realizar una propuesta más simple y transversal, ya que responde a una clasificación empírica, que forma parte del saber cotidiano de los hablantes (Ciapuscio, 1998). Por 
último, a diferencia de otras investigaciones recientes, consideramos todas las clases textuales declaradas por los profesores de las escuelas investigadas. En este sentido, no seleccionamos solo los textos considerados como paradigmáticos, como se hace en otras investigaciones (Aguilar, 2017), ni tampoco solo los de carácter jurídico, sino también otros trabajos generales de escritura, como ensayos o informes, ya que desde nuestro punto de vista también contribuyen en la formación de los futuros abogados. En consecuencia, las investigaciones anteriores a este trabajo abarcan una pequeña parte de las clases textuales que aquí consideramos. A ello hay que agregar que los textos que integran nuestra investigación han sido obtenidos a partir de lo informado por los propios docentes de dos escuelas de derecho, pertenecientes a instituciones de educación superior de distinto tipo.

Como proyección de este trabajo, en primer lugar, esperamos concretar una validación desde su implementación en espacios formativos y, de esta manera, obtener información de los agentes, tanto docentes como estudiantes, que han hecho uso de estas herramientas con el fin de conocer cuáles son las mejoras que se podrían integrar, así como los aciertos y dificultades en su diseño. En segundo lugar, pretendemos ampliar la propuesta didáctica específica que aquí planteamos a partir de la incorporación de mayor cantidad de clases textuales jurídicas.

Por último, las propuestas didácticas que presentamos se transforman en una mejor estrategia que la utilizada actualmente para producir textos propios del derecho, que es, por lo general, la copia de formatos o modelos. Asimismo, la utilización de estas experiencias didácticas podría complementarse con otras estrategias ya utilizadas por los docentes. En consecuencia, esta investigación constituye un aporte importante para los futuros profesionales de la carrera de Derecho. Así, al aplicar las propuestas ofrecidas, el alumno valorará la escritura no solo como una actividad mecánica y repetitiva, sino que podrá configurarse una representación o modelo mental en torno a la tarea de escritura, utilizando preguntas como: ¿Qué estoy escribiendo? ¿Cómo lo hago? ¿Para qué? Estas preguntas podrán ser respondidas a partir de las estrategias que aquí ofrecemos. Finalmente, no hay que olvidar que el aprendizaje implica un esfuerzo y trabajo en conjunto de sus actores, es decir, un rol activo del docente y los estudiantes. Por tanto, este diseño didáctico se transforma en un recurso que aporta tanto a la labor docente como a la formación permanente y autónoma de los abogados. Además, el valor de este recurso fue evaluado positivamente por docentes y estudiantes de derecho. 


\section{Anexo}

En esta parte del trabajo, presentamos dos anexos de la investigación. En primer lugar, en la figura 10, damos cuenta de la proporción de docentes que realizan actividades de escritura, junto a la proporción de aquellos que no lo hacen. En segundo lugar, adjuntamos la tabla 3 , en la que se presenta el perfil profesional de los docentes que forman parte de las escuelas de derecho que incluimos en la investigación.

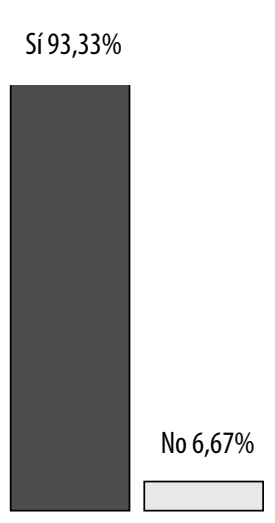

Escuela Derecho UE
Sí $97,14 \%$

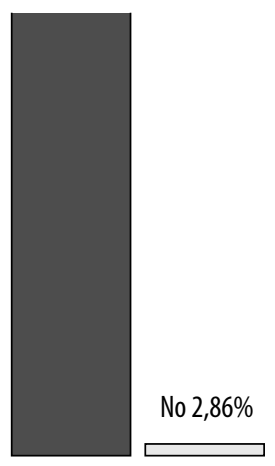

Escuela Derecho UNE

Figura 10. Proporción de docentes que realizan y no realizan actividades de escritura.

Tabla 3. Perfil profesional de los docentes de las escuelas de derecho incluidas en la investigación

\begin{tabular}{|lcccc|}
\hline & \multicolumn{2}{c}{ Universidad estatal } & \multicolumn{2}{c|}{ Universidad no estatal } \\
& Número & $\%$ & Número & $\%$ \\
Abogado o abogada & 12 & 80 & 31 & 88,57 \\
Profesora de Inglés & 0 & 0 & 1 & 2,86 \\
Profesora de Castellano & 1 & 6,67 & 1 & 2,86 \\
Ingeniero o ingeniera & 2 & 13,33 & 0 & 0 \\
Profesor de Filosofía & 0 & 0 & 1 & 2,86 \\
Enfermera & 0 & 0 & 1 & 2,86 \\
TOTAL & 15 & $100 \%$ & 35 & $100 \%$ \\
\hline
\end{tabular}

\section{Referencias}

AgüERo, Claudio (2014). « ¿Conforman las sentencias penales un género discursivo?». Estudios filológicos, 53: 7-26. DOI: 10.4067/Soo71-17132014000100001.

Aguilar, Paula (2017). «Una propuesta de géneros discursivos escritos del ámbito universitario, jurídico y chileno, orientada a la alfabetización académica de estudiantes de Derecho». Perfiles educativos, 39 (155): 179-192. DOI: 10.22201/ iisue.24486167e.2017.155.58063. 
Bautista, Nelly (2011). Proceso de la investigación cualitativa: Epistemología, metodología y aplicaciones. Bogotá: Manual Moderno.

Bisquerra, Rafael (2009). Metodología de la investigación educativa. Madrid: La Muralla.

Castelló, Montserrat (2009). «Aprender a escribir textos académicos: ¿copistas, escribas, compiladores o escritores?». En Juan Ignacio Pozo y María del Puy PérezEcheverría (coordinadores), Psicología del aprendizaje universitario: La formación en competencias (pp. 120-133). Madrid: Morata.

Ciapuscio, Guiomar (1998). «Laterminología desdeel punto de vista textual:selección, tratamiento y variación». Organon, 12 (26): 1-15. DOI: 10.22456/2238-8915.29558.

-. (2003). "Formulation and reformulation procedures in verbal interactions between experts and (semi-)laypersons». Discourse studies, 5 (2): 207-233. DOI: 10.1177/1461445603005002004.

Creswell, John y John Crewell (2017). Research Design. Qualitative, Quantitative and Mixed Methods Approaches. Londres: SAGE Publications

Cristina, Inés (2010). La deserción universitaria en primer año. Análisis de la situación de los ingresantes en la Facultad de Ciencias Naturales y Museo de La Plata a través del estudio de caso Introducción a la Botánica. Tesis de Grado, Universidad Nacional de la Plata. Disponible en bit.ly/2Cj9SBR.

Da Cunha, Iria, María Amor Montané y Luis Hysa (2017). «The arText prototype: An automatic system for writing specialized texts». En Anselmo Peñas y André Martins (editores), Proceedings of the $15^{\text {th }}$ Conference of the European Chapter of the Association for Computational Linguistics (EACL 2017). Software Demonstrations (pp. 57-60). Valencia: Association for Computational Linguistics.

Da Cunha, Iria y María Amor Montané (2020). «A corpus-based analysis of textual genres in the administration domain». Discourse Studies, 22 (1): 3-31. DOI: 10.1177/1461445619887538.

Fallahi, Carolyn, Rebecca Wood, Carol Shaw Austad y Hamid Fallahi (2009). «A Program for Improving Undergraduate Psychology Students' Basic Writing Skills». Journal Teaching of Psychology, 33 (3): 171-175. DOI: 10.1207/s15328023top3303_3.

FARFÁn, Enrique (2019). «La visión integral de la formación jurídica que surge de la retórica». Revista Pedagogía Universitaria y Didáctica del Derecho, 6 (2): 5-19. DOI: 10.5354/0719-5885.2019.36690.

González Catalán, Felipe y Karin Arismendi (2018). «Deserción estudiantil en la educación superior técnico-profesional. Explorando los factores que inciden en alumnos de primer año». Revista de la Educación Superior, 47 (188): 109-137. DOI: 10.36857/resu.2018.188.510.

GonzÁLez, Mauricio, Paulina Meza y Melissa Castellón (2019). «Medición de la Autoeficacia para la Escritura Académica: Una revisión teórico-bibliográfica». Formación Universitaria, 12 (6): 191-204. DOI: 10.4067/So718-50062019000600191. 
Graham, Steve (2018). «Introduction to Conceptualizing Writing». Educational Psychologist, 53 (4): 217-219. DOI: 10.1080/00461520.2018.1514303.

Herrera, Luis (2015). «Tipificación de apelaciones a pruebas escritas 2009-2014, Facultad de Farmacia Universidad de Costa Rica». Pensamiento Actual, 15 (25): 113120. Disponible en bit.ly/2YdHdqt.

Hyland, Ken (2013). «Faculty feedback: Perceptions and practices in L2 disciplinary writing». Journal of Second Language Writing, 22 (3): 240-253. DOI: 10.1016/j. jslw.2013.03.003.

IsENBERG, Horst (1987). «Cuestiones fundamentales de tipología textual». En Enrique Bernárdez (editor), Lingüística del Texto (pp. 95-131). Madrid: Arco Libros.

KRIPPEndORFF, Klaus (2019). «The Changing Landscape of Content Analysis: Reflections on Social Construction of Reality and Beyond». Communication \& Society, 47 (1): 1-27. Disponible en bit.ly/2YaPQlt.

LóPEZ, Sebastián (2011). «Para escribir una tesis jurídica: Técnicas de investigación en Derecho». Ius et Praxis, 17 (1): 231-246. DOI: 10.4067/So718-00122011000100010.

MAXwell, Joseph (2012). "The importance of qualitative research for causal explanation in education». Qualitative Inquiry, 18 (8): 655-661. DOI: 10.1177/1077800412452856.

Meza, Paulina y Felipe González-Catalán (en prensa). «Un instrumento para evaluar la calidad lingüístico-discursiva de textos disciplinares producidos por estudiantes de Derecho». Revista Onomázein, 51.

Meza, Paulina, Felipe González-Catalán, Carmen López-Ferrero e Israel Gutiérrez (2020). «Plain writing in the legal field: an approach from the discourse of specialists». Discourse Studies, 22 (3): 356-383. DOI: 10.1177/1461445620906027.

Moyano, Estela (2018). «La enseñanza de la lectura y la escritura académicas mediante programas a lo largo del curriculum universitario: opción teórica, didáctica y de gestión». DELTA: Documentação de Estudos em Lingüística Teórica e Aplicada, 34 (1): 235-267. DOI: 10.1590/0102-445074896274115057.

MuÑoz, Fernando (2014). «¿Hacia la academización de las facultades de Derecho en Chile? Un análisis teórico y comparado del conflicto de las profesiones». Revista de Derecho (Valdivia), 27 (1): 9-25. DOI: 10.4067/S0718-09502014000100001.

MuÑoz, Santiago (2017). Libro de estilo de la justicia. Madrid: Espasa.

OrTIZ, Elsa (2015). «Actividades y tareas de escritura académica en el contexto universitario (pregrado)». Íkala, revista de lenguaje y cultura, 20 (3): 343-358. DOI: 10.17533/udea.ikala.v2on3a05.

PARodi, Giovanni, Romualdo Ibáñez y René Venegas (2009). «El Corpus PUCV2006 del Español: identificación y definición de los géneros discursivos académicos y profesionales». Literatura y lingüística, 20: 75-101. DOI: 10.4067/ So716-58112009000100005. 
Parodi, Giovanni, René Venegas, Romualdo Ibáñez y Rosa María Gutiérrez (2015). «Géneros del Discurso en el Corpus PUCV-2006: Criterios, definiciones y ejemplos». En Giovanni Parodi (editor), Géneros Académicos y Géneros Profesionales: Accesos discursivos para saber y hacer (pp. 39-74). Valparaíso: Ediciones Universitarias Valparaíso.

PORTA, Luis y Miriam Silva (2003). «La investigación cualitativa: El análisis de contenido en la investigación educativa». Anuario digital de investigación educativa, 14: 1-18. Disponible en bit.ly/3fAlBdz.

Riquelme, Adrián, Belén Ferrer, Javier Valdés-Abellan, Miguel Ángel Pardo-Picazo, José Luis Pastor-Navarro, Miguel Cano, Roberto Tomás y Luis Jordá-Bordehore (2017). «Análisis del tiempo empleado por el alumnado de enseñanzas técnicas universitarias en pruebas escritas». En Rosabel Roig-Villa (editor), Investigación en docencia universitaria: diseñando un futuro a partir de la innovación educativa (pp. 338-397). Barcelona: Octaedro.

Rodríguez, Arturo, Jaime Espinoza, Leonardo Ramírez y Angélica Ganga (2018). «Deserción Universitaria: Nuevo Análisis Metodológico». Formación universitaria, 11 (6): 107-118. DOI: 10.4067/So718-50062018000600107.

Sologuren, Enrique, Cristina Bonifaz y Carmen Núñez (2019). «El curso basal de competencias comunicativas en Derecho: Enseñanza de la escritura académica desde un enfoque mixto e interdisciplinar». Revista Pedagogía Universitaria y Didáctica del Derecho, 6 (1): 131-154. DOI: 10.5354/0719-5885.2019.53748.

Tognini-Bonelli, Elena (2001). Corpus linguistics at work. Ámsterdam: John Benjamins.

VARGAS, Alfonso (2011). «Escribir en la universidad: reflexiones sobre el proceso de composición escrita de textos académicos». Lenguaje, 33: 97-125. Disponible en bit.ly/37EqfEx.

Villarroel, Verónica, David Boud, Susan Bloxham, Daniela Bruna y Carola Bruna (2019). «Using principles of authentic assessment to redesign written examinations and tests». Innovations in Education and Teaching International, 57 (1): 38-49. DOI: 10.1080/14703297.2018.1564882.

Villarroel, Verónica, Daniela Bruna, Claudio Bustos, Carola Bruna y Carolina Márquez (2018). «Written tests analysis under the principles of authentic assessment. A comparative study of written tests of medical and other undergraduate programs». Revista Médica de Chile, 146 (1): 46-52. DOI: 10.4067/so034-98872018000100046.

\section{Financiamiento}

Esta investigación fue posible gracias al financiamiento del Proyecto Fondecyt de Iniciación 11170128 titulado «Caracterización discursiva de géneros producidos por estudiantes de derecho y medicina: Su relación con la percepción de la autoeficacia 
en la escritura, la calidad general del texto y la evaluación disciplinar». Por otra parte, también fue importante el apoyo de la Dirección de Investigación de la Universidad de La Serena.

\section{Agradecimientos}

Agradecemos la valiosa colaboración de los expertos que participaron en los distintos procesos de validación incluidos en esta investigación, específicamente, a los abogados Israel Gutiérrez de la Universidad Católica del Norte y Andrea Pinto de la Pontificia Universidad Católica de Valparaíso; y a los estudiantes de derecho de la Pontificia Universidad Católica de Valparaíso: Enzo Buglioni Calderón, Sebastián Mesa Crocco, Franco Muñoz Sepúlveda y Francisca Ugalde Sandoval.

\section{Sobre los autores}

Paulina Meza es profesora de Castellano y licenciada en Educación por la Universidad de Playa Ancha. Además, es magíster en Lingüística Aplicada y doctora en Lingüística por la Pontificia Universidad Católica de Valparaíso. A partir de su investigación postdoctoral, se especializó en escritura científica. Su correo electrónico es pmeza@userena.cl. (D) https://orcid.org/oooo-ooo1-8300-9681.

Felipe González-Catalán es profesor de Castellano y Comunicación, licenciado en Educación, y licenciado en Lengua y Literatura Hispánica por la Pontificia Universidad Católica de Valparaíso. Además, es magíster en Educación con mención en Evaluación de Aprendizajes por la Pontificia Universidad Católica de Chile y doctor (c) en Educación y Sociedad por la Universidad de Barcelona. Su correo electrónico es felipe.gonzalez.c@pucv.cl. (D) https://orcid.org/oooo-0003-1153-1723.

Allison Pastén es licenciada en Ciencias Jurídicas por la Universidad Católica del Norte. Su correo electrónico es allison.pasten@gmail.com. (D) https://orcid. org/0000-0002-7111-4473.

Maximiliano Barahona es estudiante de licenciatura en Ciencias Jurídicas en la Pontificia Universidad Católica de Valparaíso. Su correo electrónico es maximiliano. barahona.v@mail.pucv.cl. (D) https://orcid.org/oooo-0oo2-9150-4006. 
La Revista Pedagogía Universitaria y Didáctica del Derecho (RPUDD) es una publicación científica semestral que contribuye a la reflexión multidisciplinaria sobre pedagogía universitaria y didáctica del derecho, para la formación y consolidación de esta área de investigación; así como a la difusión de prácticas innovadoras en la enseñanza-aprendizaje del derecho considerando el contexto nacional e internacional. Es una publicación electrónica internacional con una codirección entre Brasil y Chile.

\author{
DIRECTORA \\ María Francisca Elgueta Rosas \\ Universidad de Chile \\ DIRECTOR \\ Renato Duro Dias \\ Universidad Federal de Rio Grande, Brasil \\ SITIO WEB \\ pedagogiaderecho.uchile.cl \\ CORREO ELECTRÓNICO \\ rpedagogia@derecho.uchile.cl \\ LICENCIA DE ESTE ARTÍ́CULO
}

Creative Commons Atribución Compartir Igual 4.0 Internacional

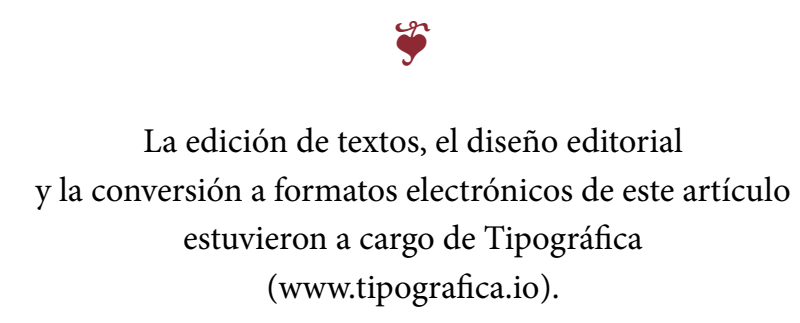

